\title{
CINCUENTA AÑOS (50) DE ENFERMEDAD PELVICA INFLAMATORIA EN EL HOSPITAL SAN JUAN DE DIOS - BOGOTA
}

\author{
Dr. Alvaro Fonnegra Miramón* - Dr. Rubén Darío Guzmán A.**
}

\author{
TRABAJO PRESENTADO AL VI CONGRESO DE OBSTETRICIA Y GINECOLOGIA POR LA SECCION \\ DE OBSTETRICIA Y GINECOLOGIA DE LA FACULTAD DE MEDICINA DE LA \\ UNIVERSIDAD NACIONAL DE COLOMBIA. \\ Hospital San Juan de Dios - Bogotá, D. E.
}

Hemos querido presentar una revisión de la enfermedad pélvica inflamatoria (*) en el Hospital San Juan de Dios de Bogotá, D. E., que abarca una época comprendida entre 1908 y 1965 o sea cincuenta y siete años. Se exceptúan siete años entre 1935 y 1942, pues desafortunadamente la estadística de ese período se extravió, lo que da un total de cincuenta años.

De acuerdo con una estadística del Ministerio de Salud sobre morbilidad en Colombia, el Hospital San Juan de Dios de Bogotá, atiende actualmente el $10 \%$ de la población enferma del país.

Como recuento histórico el Hospital San Juan de Dios funcionó en un edificio de la Carrera 9: entre Calles 11 y 12 de Bogotá, desde mediados de la Colonia, hasta el año de 1926,

\footnotetext{
* Profesor Asociado.

** Instructor.
}

(*) Con este nombre se denominan en la actualidad, todas las entidades inflamatorias e infecciosas, de tipo ginecológico, como parametritis, salpingitis, anexitis, pelviperitonitis, etc., sea cual sea su etiología y estado clínico). época en que se trasladó al sitio llamado de "La Hortúa", donde actualmente se encuentra.

Afortunadamente los datos del archivo de la clínica ginecológica, dirigidos por el Profesor Rafael Ucrós padre de la ginecología en Colombia, se conservan intactos desde 1908 hasta 1935.

En dichos documentos se puede apreciar la forma ordenada y precisa como se llevaban las historias clínicas en ese entonces. Se puede decir que no hay dato, por pequeño que sea, que no se encuentre consignado en el historial clínico.

En consecuencia no podemos menos de rendir un homenaje de admiración y reconocimiento a la labor paciente desarrollada por todos los médicos que intervinieron en esa etapa del desenvolvimiento de la Ginecología, en nuestro medio.

Para nuestro estudio hemos dividido en tres (3) etapas fundamentales el análisis de las historias clínicas:

Primera etapa: Comprende desde 1908 hasta 1935 o sea hasta el advenimiento de las sulfas. 
Segunda etapa: Desde 1942 hasta 1946 época de la Sulfamidoterapia.

Tercera etapa: 1947 hasta 1965, tiempo del uso de la Penicilina y demás antibióticos descubiertos con posterioridad.

CUADRO NN 1

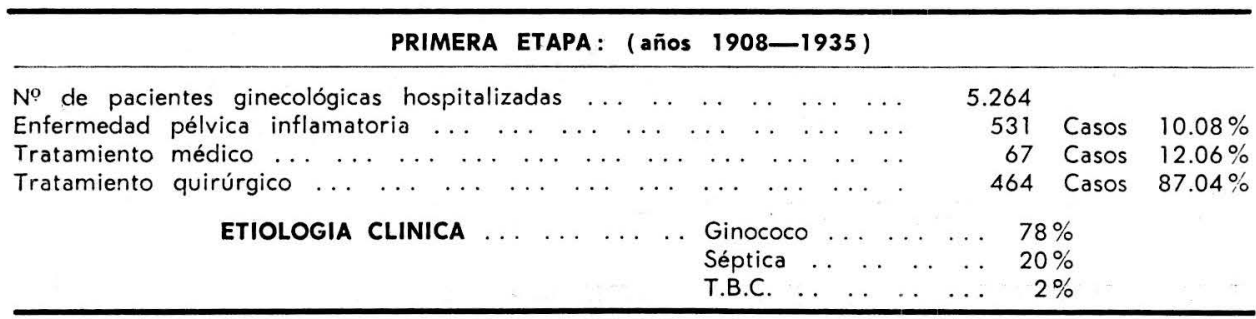

Primera etapa: Se analizaron 5.264 casos ginecológicos de pacientes hospitalizadas de las cuales se encontraron 531 casos de enfermedad pélvica inflamatoria, la mayoría comprendidas bajo el diagnóstico de anexitis $y$ abscesos pélvicos, lo que da un porcentaje global de $10.08 \%$ en relación con las demás entidades ginecológicas.

De éstas 531 pacientes, 464 o sea un $87,4 \%$ fueron llevadas a cirugía y el resto o sea 67 , para un $12,6 \%$ recibieron tratamiento médico.

La etiología clínica posible era el gonococo en un $78 \%$, contra un $20 \%$ de etiología séptica o piógena y un $2 \%$ para la TBC.

El tratamiento médico en esa época consistía principalmente en:

Reposo absoluto, hielo, desinfectantes generales (Urotropina, salol), grandes irrigaciones vaginales calientes (Precursoras de la diatermia) con sol. de Permanganato o azul de Metileno, óvulos de ictiol y argirol, laxantes y purgantes.

Si después de un corto período de medicación, la enferma no mejoraba, como usualmente ocurría, se intervenía quirúrgicamente y sin tener en cuenta la edad ni la paridad, se practicaba casi de rutina la histerecto- mía sub-total con salpingo-ooforectomía bilateral. Solamente en los casos incipientes de salpingitis agudas, se hacía cirugía conservativa en pacientes jóvenes.

Para la T.B.C. bastaba con la sola laparotomía o aplicación de lámpara de cuarzo. En ocasiones se lavaba con éter la cavidad abdominal.

La anestesia empleada al principio fue el cloroformo en la mayoría de las pacientes. Posteriormente llegó el éter empleándose éste solo o en mezcla de Schleich.

Exámenes paraclínicos, de laboratorio e histopatología.

Las pielografías comenzaron a aparecer como exámenes rutinarios en 1930. Así mismo la histopatología y la fotografía de piezas macroscópicas, desde 1927.

Los parciales de orina desde 1925; exámenes de flujos (directo y por coloración) desde 1930.

Con el advenimiento del leucograma en 1929 y la sedimentación globular en 1934, como exámenes de rutina, en los casos de enfermedad pélvica inflamatoria, se marca un jalón importantísimo en el diagnóstico, evolución, pronóstico y control de esas entidades. A propósito de controles, éstos eran muy escasos. 
CUADRO NN 2

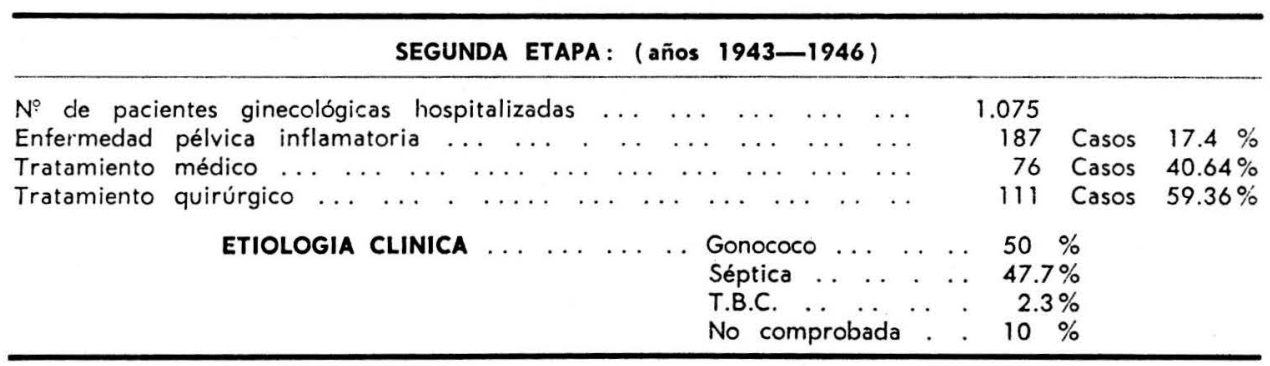

Segunda etapa: Comprende desde 1943 hasta 1946, tiempo en que hacen su aparición la sulfamidoterapia, comenzando con el Prontosil rojo y posteriormente con toda la variedad de clerivados que hoy existen en la terapéutica.

El Profesor Hernando Amaya León analiza en su tesis de grado de 1944, entre otros temas, el de Enfermedad Pélvica Inflamatoria y posteriormente el Profesor Alvaro Espinosa y Espinosa, también en su tesis de grado en 1946, comenta de una manera exhaustiva este tópico. De estas dos tesis de grado extractamos los principales aspectos de la Enfermedad Pélvica Inflamatoria en ese tiempo.

De 1.075 enfermas ginecológicas hospitalizadas en ese lapso aparecieron 187 historias de Enfermedad Pélvica Inflamatoria lo que da un porcentaje de $17.4 \%$, que muestra claramente un aumento de la incidencia en relación con la primera etapa, crecimiento que podría explicarse por las reinfecciones en tratamientos médicos incompletos o mal conducidos ya que, como antes decimos, el tratamiento era primordialmente quirúrgico mutilante, sin posibilidades por consiguiente de recidiva.

En combinación con las sulfas se utilizaba, para las fases crónicas, la vacunoterapia inespecífica con resultados relativos. La diatermia, como coadyuvante, con resultados favorables más definidos.

Por esa época se incrementó el tratamiento médico contra lo que se venía haciendo, llegando a la cifra de un $40.64 \%$. Con la introducción de la sulfamidoterapia se notó la racionalización en el tratamiento de la Enfermedad Pélvica Inflamatoria.

Cuando se practicaba cirugía, la operación de elección consistía en la Salpinguectomía uni o bilateral y en pocas ocasiones la histerectomía. Los ovarios no se extirpaban a menos que existiese una patología definida en ellos, procurando siempre conservar uno de los dos, sobre todo en mujer joven.

El porcentaje de tratamiento quirúrgico fué de $59.36 \%$.

La complicación de peritonitis debida generalmente a abscesos tuboováricos estallados o a maniobras abortivas con perforación uterina, se trataban por evacuación del pus y drenaje. Tenía una mortalidad casi del $100 \%$. En cuanto a la pelviperitonitis se trataba con colpotomía además del tratamiento médico sub-siguiente.

Tercera etapa: Se inicia con la era de la penicilina introducida en los servicios hospitalarios en forma paulatina y posteriormente de manera rutinaria. Cubre desde 1946 hasta junio de 1965 o sea un período de 18 años y medio. 


\section{CUADRO № 3}

TERCERA ETAPA: (años 1946 a Junio/65)

INCIDENCIA $Y$ TRATAMIENTO

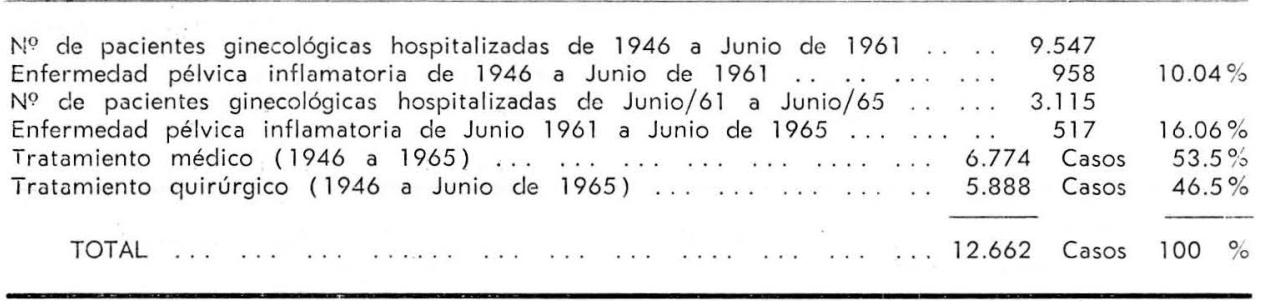

CUADRO NN 4

TERCERA ETAPA: (años 1946 a Junio/65) ETIOLOGIA

AÑOS $(1946-1961)$

JUNIO 1961 A JUNIO 1965

\begin{tabular}{|c|c|c|c|}
\hline onococo & & & $34.7 \%$ \\
\hline$\ldots \quad \ldots$ & $\ldots$ & $\ldots \quad \ldots$ & $44.5 \%$ \\
\hline T. B. C. . . & . . . & . . . . & $5.1 \%$ \\
\hline No comprobada ... & $\ldots \quad \ldots$ & $\ldots \ldots$ & $15.7 \%$ \\
\hline Gonococo .. & $\ldots \ldots$ & $\ldots \ldots$ & 35 \\
\hline$\ldots \quad \ldots \quad \ldots$ & $\ldots \ldots$ & $\ldots \ldots$ & 45 \\
\hline B. C. . . . . . & 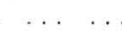 & & 20 \\
\hline
\end{tabular}
$5.1 \%$
Se hospitalizaron 12.662 casos ginecológicos, con 1.475 casos d) Enfermedad Pélvica Inflamatoria lo que da un promedio global de $10.03 \%$.

Posteriormente a la penicilina aparecieron los demás antibióticos de amplio espectro (Cloranfenicol, Tetraciclinas, etc.).

Se incrementa todavía más el tratamiento de las infecciones pélvicas, combinando las sulfas ya muy perfeccionadas con la Penicilina y demás medidas generales.

La Penicilina se usa como arma de choque inicial y solamente por medio de cultivos y antibiogramas se cambia, si es necesario, por el antibiótico de elección.

Hacia el año de 1955 un Profesor del servicio de Ginecología y Obstetricia, el Dr. Jorge Amorocho Carreño, presenta un trabajo sobre el uso do los antiflogísticos tipo Fenil-butazona en las afecciones anexo-parametriales y su aplicación se rutiniza en el servicio de Ginecología.

Se continúan empleando las vacunas y diatermia para los casos crónicos con los resultados ya mencionados y la cirugía se vuelve electiva para aquellos casos, donde el suficiente tratamiento médico no logra reducir las masas pélvicas. 
CUADRO $N$ N 5

\begin{tabular}{lcccc}
\hline & N. de casos & Morbilidad & Mortalidad \\
\hline LAVADO PERITONEAL & 70 & $50 \%$ & 5 Casos $7.1 \%$ \\
SIN LAVADO PERITONEAL & 30 & $70 \%$ & 6 Casos $20 \%$ \\
\hline
\end{tabular}

Hacia mediados de 1962 se comenzó a emplear el método quirúrgico del lavado peritoneal, para los casos de peritonitis generalizadas y Shock bacteriémico de origen ginecológico y obstétrico. Con dicho sistema se logró reducir la mortalidad, que para ese entonces era de un $20 \%$, a un $7.1 \%$.

En esta tercera etapa se ha tratado médicamente de acuerdo con los Cuadros, de 1946 al $65,53,6 \%$ y quirúrgicamente el $46.4 \%$. Como se nota hay un incremento en el tratamiento médico.

En una forma ostensible se ve disminuir la incidencia de Enfermedad Pélvica Inflamatoria en general, que de un $17,4 \%$ sobre el total de casos ginecológicos hospitalizados en 1946 baja a un $10.04 \%$ hacia 1961. Sin embargo a partir de esta fecha vuelve a subir y para Junio de 1965 tenemos un $16.06 \%$.

Si analizamos las tres etapas y las correlacionamos entre sí, vemos detalles sumamente importantes en re- lación con la etiología, diagnóstico y evolución de la Enfermedad Pélvica Inflamatoria.

Así la primera, en la etiología clínica figuraba con un $78 \%$ el gonococo, para un $20 \%$ los gérmenes piógenos o sépticos (Tipo estáfilo-estreptococo-colibacilo) y la T.B.C. con un $2 \%$.

Ya para 1945 nos da una cifra del $50 \%$ para gonococo, $37.7 \%$ para gérmenes piógenos y $2.3 \%$ para la T.B.C. sin comprobación en el 10\%.

Hacia 1961 las cifras se invierten fundamentalmente dando el siguiente resultado:

$34.7 \%$ para la etiología Neisseriana y $44.5 \%$ para la infección séptica o piógena. $5.1 \%$ para la T.B.C. y el resto o sea un $15.7 \%$ sin etiología comprobada.

De 1961 a Junio de 1965 el gonococo se encuentra en un $35 \%$, la septis en un $45 \%$ y T.B.C. para un $20 \%$.

\section{CUADRO № 6}

\section{INCIDENCIA DE PERITONITIS POR AÑOS}

\begin{tabular}{|c|c|c|c|c|c|c|c|c|c|c|c|c|c|c|c|c|c|}
\hline$D E$ & MARZO & $D E$ & 1959 & a & MARZO & $\mathrm{DE}$ & 1960 & $\ldots$ & & $\cdots$ & $\cdots$ & $\cdots$ & & & & . & 6 \\
\hline DE & MARZO & $D E$ & 1960 & a & MARZO & $\mathrm{DE}$ & 1961 & $\ldots$ & . & $\cdots$ & $\cdots$ & $\ldots$ & $\cdots$ & $\cdots$ & $\cdots$ & $\ldots$ & 6 \\
\hline $\mathrm{DE}$ & MARZO & $\mathrm{DE}$ & 1961 & a & MARZO & $\mathrm{DE}$ & 1962 & $\ldots$ & $\ldots$ & $\ldots$ & $\ldots$ & $\ldots$ & $\ldots$ & $\ldots$ & $\ldots$ & $\ldots$ & 7 \\
\hline DE & MARZO & DE & 1962 & a & MARZO & $\mathrm{DE}$ & 1963 & $\ldots$ & . . & $\ldots$ & .. & $\cdots$. &. & $\ldots$ & $\ldots$ & . & 15 \\
\hline$D E$ & MARZO & $\mathrm{DE}$ & 1963 & a & MARZO & $\mathrm{DE}$ & 1964 & $\ldots$ & $\ldots$ & $\ldots$ & . & $\ldots$ & $\ldots$ & $\ldots$ & $\ldots$ & . & 16 \\
\hline $\mathrm{DE}$ & MARZO & $\mathrm{DE}$ & 1964 & a & MARZO & $\mathrm{DE}$ & 1965 & $\ldots$ & $\ldots$ & $\ldots$ & $\ldots$ & $\ldots$ & $\ldots$ & $\ldots$ & $\ldots$ & $\ldots$ & 28 \\
\hline
\end{tabular}


Por otra parte vemos en el cuadro correspondiente la incidencia de peritonitis, un franco aumento de esta entidad de 1959 a 1965, lo que demuestra una virulencia mayor de los gérmenes, posiblemente por resistencia microbiana a los antibióticos, por su uso indiscriminado y por otra par- te aumento de la incidencia de abortos provocados de tipo criminal. Si esto sucede en la Unidad Ginecológica, con mayor razón en los Servicios Obstétricos donde las cifras de abortos han aumentado en forma alarmante.

\section{CUADRO N: 7}

INCIDENCIA DE GERMENES DE ACUERDO CON SU FRECUENCIA OBTENIDOS DE PUS PROCEDENTE DE ABSCESOS INTRAPERITONEALES

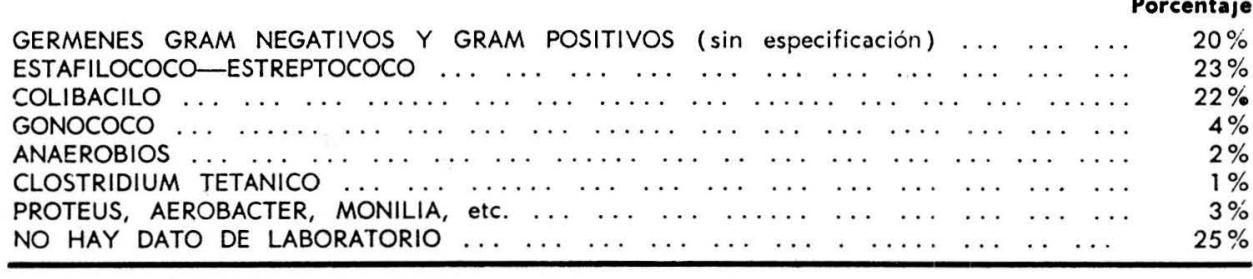

En lo que hace relación al gonococo, éste parece ser un germen sumergido, es decir que no se hace aparente con los medios de laboratorio usuales, motivo por el cual no se encuentra sino en una muy pequeña proporción, tanto en las secreciones vulvo-vagino-cervicales como en abscesos pélvicos extirpados quirúrgicamente. Se necesitan métodos de laboratorios más precisos y fieles para detectarlo, como por ejemplo el procedimiento de la inmuno-fluorescencia, que no se emplea todavía en nuestro medio.

La T.B.C. también aumentó su incidencia pues del $2 \%$ que se diagnosticaba en la primera etapa, ascendió a $5.1 \%$ hacia 1961. De esa parte en adelante y gracias a los métodos más perfeccionados de laboratorio para la pesquiza del bacilo, a las biopsias de endometrio rutinarias, y a las Historias Clínicas más completas, llegó hasta el $20 \%$ en Junio de
1965, como lo ha comprobado Amaya León, Vergara Támara y Fonnegra Miramón.

\section{RESUMEN $Y$ CONCLUSIONES}

1. Se hace un análisis exhaustivo de la enfermedad pélvica inflamatoria en el Hospital San Juan de Dios de Bogotá, en un lapso de 50 años.

2. En ese período se hospitalizaron 19.001 pacientes ginecológicas $y$ se presentaron 1.988 casos de E. P. I. para un $10.9 \%$.

3. Se divide en tres (3) etapas el estudio de esta revisión, que comprenden:

a) De 1908 a 1935, donde predominó el tratamiento quirúrgico.

b) De 1943 a 1946 época de la sulfamidoterapia en donde se incrementó el tratamiento médico, pero aún era mayor la proporción de tratamientos quirúrgicos. 
CUADRO № 8

\begin{tabular}{|c|c|c|c|c|c|c|c|}
\hline \multicolumn{8}{|c|}{ RESUMEN DE CONDUCTAS } \\
\hline & T. médico & T. quirúrgico & Mejoría & Recidivas & $\begin{array}{c}\text { Curación } \\
\text { clínica }\end{array}$ & Controles & Mortalidad \\
\hline SALPINGITIS y Anexitis agudas & TODAS & & $100 \%$ & $30 \%$ & $70 \%$ & $30 \%$ & \\
\hline $\begin{array}{l}\text { PELVIPERITONITIS con Abscesos locali- } \\
\text { zados al Douglas }\end{array}$ & TODAS & $\begin{array}{l}\text { Colpotomía } \\
\text { Laparotomía }\end{array}$ & $\begin{array}{l}60 \% \\
40 \%\end{array}$ & & $98 \%$ & $30 \%$ & $2 \%$ \\
\hline $\begin{array}{l}\text { MASAS ANEXIALES definidas crónicas. } \\
\text { PIOSALPINX y abscesos tubo-ováricos } \\
\text { encapsulados }\end{array}$ & TODAS & Laparotomía & $\begin{array}{l}45 \% \\
55 \%\end{array}$ & & $99 \%$ & $30 \%$ & $1 \%$ \\
\hline
\end{tabular}

PIOSALPINX y abscesos tubo-ováricos estallados $Y / 0$ PERFORACIONES UTERINAS
TODAS LAVADO PERITONEAL: Paciente joven: S. O. Unilateral y salpingectomía contralateral.

Con sepsis endouterina: Histerectomía con Salpingo-ooforectomía bilateral.

Paciente pre- o post-menopausia: Histerectomía con S.O.B.

\section{CUADRO № 9}

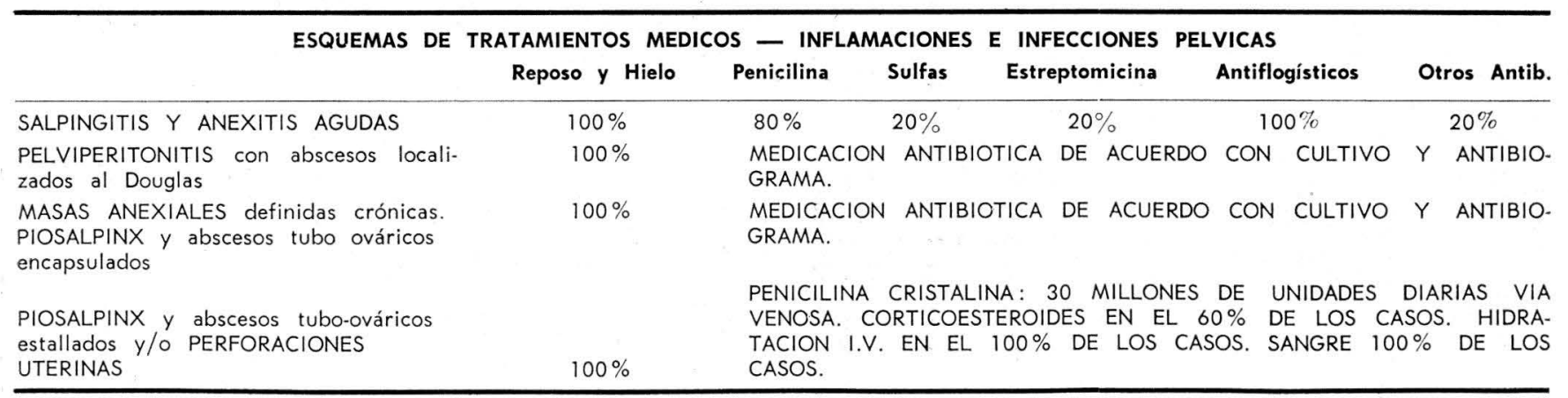


La incidencia de Enfermedad Pélvica Inflamatoria en nuestro medio, que comenzó siendo de un $10.08 \%$ entre 1908 y 1935, sufre una alza para 1946 de $17.4 \%$, vuelve a descender hacia 1961 a un $10.04 \%$ y nuevamente ascender hacia Junio de 1965 a un $16.06 \%$.

4. El Gonococo es un germen muy difícil de detectar por los medios usuales de laboratorio.

5. Los gérmenes sépticos o piógenos tipo (estáfilo-estreptococo-colibacilo) han aumentado considerablemente, y esto se explica por el aumento alarmante de los abortos criminales hechos sin ninguna asepsia, y la resistencia microbiana por el uso indiscriminado de los antibióticos.

6. La incidencia de E. P. I. por T.B. C. ha aumentado considerablemente en los últimos cuatro (4) años, pero interpretamos este aumento no por mayor contagio de las pacientes sino por el avance en los métodos de diagnóstico.

7. Las pelviperitonitis, las peritonitis generalizadas y el Shock bacteriémico, como complicación de infección ginecológica han aumentado también en forma progresiva, por las mismas razones expuestas en el punto $5^{\circ}$. de estas conclusiones.

Para terminar deseamos preguntar a la distinguida concurrencia de colegas a este Congreso, lo siguiente:

1. La prostitución en Colombia debe prohibirse, o por el contrario debe reglamentarse y controlarse en forma técnica?

2 . Se debe implantar en nuestro medio el control de la natalidad, para regular la población, o debemos permanecer indiferentes al aumento escandaloso de los abortos criminales con sus fatales consecuencias?

\section{BIBLIOGRAFIA}

1 AMAYA LEON HERNANDO: Comentario a un año de Internado. Tesis de Grado. Universidad Nacional, 1944.

2 AMOROCHO CARREÑO JORGE: Nuevas anotaciones sobre el uso de la fenilbutazona en las obstrucciones tubarias. Rev. Col. Obst. y Gynec. Vol. II. No 3, 1960.

3 APARICIO RUEDA ALBERTO: La C. R. P. (reactivo protein) como auxiliar en el diagnóstico de apendicopatías e infecciones anexiales. Tesis de grado. Rev. Col. Obs. y Ginec. Vol. 8, № 6, 1957

4 CRUZ AMAYA ARISTOBULO: Dos casos de piosalpinx tuberculoso. Rev. Col. Obst. y Ginec. Vol. II, № 5, 1960.

5 ESPINOSA Y ESPINOSA ALVARO: Anexitis, comentarios a 3 años de estadística en el Servicio de Ginecología del Hospital San Juan de Dios. Tesis de Grado. Univ. Nacional, 1946.

6 DE ALVAREZ, RUSSELL R., FIGGE DAVID C.: Influence of antibiotics en pelvic inflamatory disease. Obst. y Ginec. N. Y. Vol. 5, № 6, Junio, 1955.

$7 \mathrm{H}$. B. ATLLE: Acute Gynecological and Obstetrical Emergencies. The Surgical Clinics of North America. Vol. 40, № 5, octubre, 1960.

8 BAYLY NELVYN A., GENTLIN ALLWYN H.: Residues of pelvic inflamatory disease and abnormal uterine bleeding. Am. J. Obst. y Gynec. Vol. 82, № 3, septiembre, 1961.

9 BLINICK GEORGE: Gonorrheal disease in the female. Clinical Obst. \& Gynec., June, 1959.

10 CHATTAS ALBERTO: Eritrosedimentación. Su aplicación a la clínica. Salvat Edtores, 1943.

11 CHEVALIER RAUL, SANMARTINO R.: Permeabilidad tubárica y T.B.C. Obst. \& Ginec. Lat. Am. IX: 450, 1951.

12 COLLINS CONRAD G., NIX FRANK G. and CERHA HARRY T.: Ruptured tuboovarian abscess. Am. J. Obst. \& Gynec. Vol. 72, No 4, octubre, 1956.

13 COLLINS CONHAR G., JANSEN FREDERICK: Tratamiento de los abscesos pélvicos. Clin. Obst. \& Gynec. Pág. 512, junio, 1959.

14 DOUGLAS GILBERT F. et al.: Pelvic pain in women. The J. of Int. Coll. of Surgeons. Vol. 35, № 1, enero, 1961. 
15 HALBRETCH I.: Tuberculosis genital. Ginec. Prat. 9: 419, 1958.

16 KARTER E., RUMMO B. P.: Surgical bleeding tendencies of patients with chronic pelvic inflamatory disease. Am. J. Obst. \& Gynec. Vol. 75, No 1.100, 1957.

17 KURLAND IRVING I., LOUGHRAN CHARLES $\mathrm{H}$.: Corticosteroids in the treatment of nonpatent fallopian tubes. Am. J. Obst. \& Gynec. Vol. 81, N. 2, febrero, 1961.

18 MEIGS and STURGIS: Progress in Gynecology. Vol. II .Pág. 402. Grunne \& Stratton, 1958.

19 MIALE JOHN B.: Laboratory Medicine. Hematology. The C. V. Mosby Company St. Louis, 1958.

20 NOVAK and JONES: Texbook of Gynecology, 1964.

21 REACH ERNEST: Las ondas cortas en Ginecología. Editorial Pegaso. Madrid 1942.

22 ROBISION S. C.: Pelvic abscess. Am. J. Obst. \& Gynec. Vol. 81, No 2, febrero, 1961.

23 SCHAEFER GEORGE: Antituberculous treat- ment of tuberculous salpingitis. Antimicrobial. Am. J. Obst. \& Gynec. Vol. 77: 996, 1959.

24 TRITSCH JOHN E., SAXE IRVING H., SCHNEDER E.: Pelvic inflamatory disease. A surgery of 300 consecutive cases, with special reference to treatment. The New England J. of Med. Vol. 235, No 12, Sept. 19, 1946.

25 WALKER VERN L., BAKER W. S. Jr.: Surgical management of acute pelvic infection refractory to conservative therapy. Am. J. Obst. \& Gynec. Vol. 82, № 3, septiembre, 1961.

26 WOOTEN ERNEST L.: Use of posterior colpotomy in the diagnosis and treatment of ectopic pregnancy and other lesions of the pelvic. Am. J. Obst. \& Gynec, Vol. 80, N 4, octubre, 1960.

27 ESPINOSA ESPINOSA ALVARO, HANDZER BERNARDO, ALBA LUIS: Enfermedad pélvica inflamatoria. Comentario a dos años y medio de estadística en el servicio de Ginecología. Hospital San Juan de Dios Bogotá. Revista Col. de Obs. y Ginec. Vol. XII, No 6. Nov-Dic. 1961, Págs. 532-555. 